$-140(0 \cdot 8)^{\circ}(\mathrm{I}), 54(1)^{\circ}$ (II)]. It seems that there is a certain degree of flexibility at each end of the molecule with the cyclopropane carboxylic linkage in the middle forming a fairly rigid entity. No intermolecular distances are less than the sum of the van der Waals radii of the atoms involved, except that between $\mathrm{C}(7)-\mathrm{C}(20)=3.52 \AA(x, y, 1+z)$. The lack of close intermolecular contacts (no hydrogen bonds) in the crystal structures so far determined is certainly related to the great solubility of those compounds in water.

\section{References}

Germain, G., Main, P. \& Woolfson, M. M. (1971). Acta Cryst. A27, 368-376.

SHELDRICK, G. M. (1976). SHELX76. Program for crystal structure determination. Univ. of Cambridge, England.

Stewart, R. F., Davidson, E. R. \& Simpson, W. T. (1965). Chem. Phys. 42, 3175-3187.

TESSIER, J. (1985). Recent Advances in the Chemistry of Insect Control, edited by N. F. JANES, pp. 26-52.

Tessier, J., Teche, A. \& Demoute, J. P. (1983). Pesticide Chemistxy, Human Welfare and the Environment, Vol. I, edited by J. Myamoto \& P. C. Kearney, pp. 95-100. Oxford: Pergamon Press.

Acta Cryst. (1991). C47, 771-775

\title{
Structures of Two Conformationally Defined Phenylethanolamines: exo-1,4-Epoxy-2-formamido-1,2,3,4-tetrahydro-8-trifluoromethylnaphthalene and exo-1,4-Epoxy-2-formamido-1,2,3,4-tetrahydro-6-trifluoromethylnaphthalene
}

\author{
By Gary L. Grunewald* and Moorthy S. S. Palanki \\ Department of Medicinal Chemistry, The University of Kansas, Lawrence, KS 66045, USA \\ AND Fusao TAKusagawa \\ Department of Chemistry, The University of Kansas, Lawrence, KS 66045, USA
}

(Received 3 April 1989; accepted 25 June 1990)

\begin{abstract}
Epoxy-2-formamido-1,2,3,4tetrahydro-8-trifluoromethylnaphthalene $\mathrm{C}_{12} \mathrm{H}_{10} \mathrm{~F}_{3} \mathrm{NO}_{2}, M_{r}=257 \cdot 21$, Pccn, $a=8.895(1), b=$ $19.968(5), c=12.656(3) \AA, V=2247.9(8) \AA^{3}, Z=$ $8, D_{x}=1.520 \mathrm{~g} \mathrm{~cm}^{-3}, \lambda($ Mo $K \alpha)=0.71069 \AA, \mu=$ $1.49 \mathrm{~cm}^{-1}, F(000)=1056, T=297 \mathrm{~K}, R=0.0466$ for 1481 independent reflections collected. The torsion angle for $\mathrm{N}(10)-\mathrm{C}(2)-\mathrm{C}(1)-\mathrm{C}(8 a)$ is $167 \cdot 4(2)^{\circ}$. ( \pm )-exo-1,4-Epoxy-2-formamido-1,2,3,4-tetrahydro6-trifluoromethylnaphthalene, (2), $\mathrm{C}_{12} \mathrm{H}_{10} \mathrm{~F}_{3} \mathrm{NO}_{2}, M_{r}$ $=257.21, \quad P 2_{1} 2_{1} 2_{1}, a=8.52(2), b=26 \cdot 15(2), c=$ 5.06 (5) $\AA, \quad V=1127(11) \AA^{3}, \quad Z=4, \quad D_{x}=$

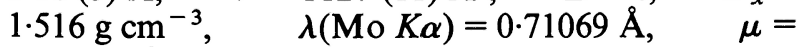
$1.29 \mathrm{~cm}^{-1}, F(000)=528, T=297 \mathrm{~K}, R=0.108$ for 932 independent reflections collected. The torsion angle $\mathrm{N}(10)-\mathrm{C}(2)-\mathrm{C}(1)-\mathrm{C}(8 \mathrm{a})$ is $172.5(6)^{\circ}$; the formamido group in both (1) and (2) is exo. X-ray studies on (1) suggest a hydrogen bond between $\mathrm{N}(10)$ and $\mathrm{O}(12)$ and similarly for (2).
\end{abstract}

Introduction. As part of our studies to understand the relationship between conformations of biogenic amines and their affinities for the active site of phenylethanolamine $N$-methyltransferase (PNMT)

* To whom correspondence should be addressed.

0108-2701/91/040771-05\$03.00
(Grunewald, Arrington, Bartlett, Reitz \& Sall, 1986; Grunewald, Ye \& Takusagawa, 1987; Ye \& Grunewald, 1989), we have prepared ( \pm )-exo-1,4epoxy-2-formamido-1,2,3,4-tetrahydro-8-trifluoromethylnaphthalene $(1)$ and ( \pm )-exo-1,4-epoxy2-formamide-1,2,3,4-tetrahydro-6-trifluoromethylnaphthalene (2). These are conformationally defined analogs of trifluoromethyl-substituted $\beta$-phenylethanolamine, in which the ethanolamine side chain has been fixed.

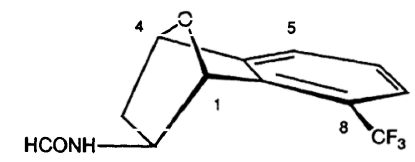

(1)

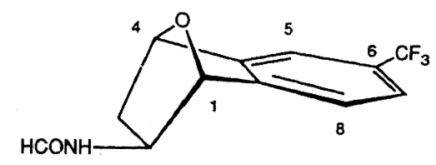

(2)

In the case of the analogous compounds with a methylene bridge replacing the oxo bridge

(C) 1991 International Union of Crystallography 
(Grunewald, Paradkar, Pazhenchevsky, Pleiss, Sall, Seibel \& Reitz, 1983), addition of diborane to olefin (3) $\left(X=-\mathrm{CH}_{2}-\right)$ followed by oxidation and conversion to the amine produced 5-substituted and 8-substituted analogs (4) and (5) $\left(X=-\mathrm{CH}_{2}-\right)$ in a $1: 1$ ratio. Azido mercuration of olefin (3) $(X=$ $-0-)$ followed by reduction produced amines (4) and (5) $(X=-\mathrm{O}-)$ in a ratio of $1: 9$ with $(5)(X=$ $\left.-\mathrm{O}^{-}\right)$as the major isomer. Spectroscopic information on the major isomer was consistent with structure (5) but was not unambiguous. In order to interpret the biochemical data of the interaction of (4) and (5) $\left(X=-\mathrm{CH}_{2}-\right.$ and $\left.X=-\mathrm{O}-\right)$ with PNMT, it was essential to have unambiguous structural assignments. For this reason the X-ray crystal structure of the formamide derivative of $(5)(X=$ $-\mathrm{O}_{-}$), compound (1), was determined. Compounds (6) and (7) were later prepared to provide the other two possible regioisomers of trifluoromethyl substitution and, to differentiate them unambiguously, the $\mathrm{X}$-ray crystal structure of the formamide derivative of (6), compound (2), was also determined.

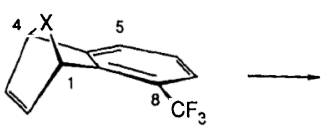

(3)

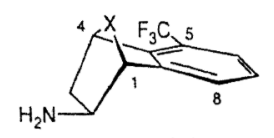

(4)

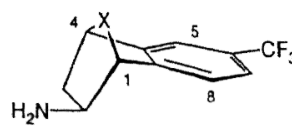

(6)

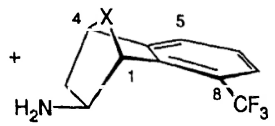

(5)

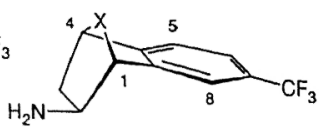

(7)
Experimental. exo-1,4-Epoxy-2-formamido-1,2,3,4tetrahydro-8-trifluoromethylnaphthalene (1) was obtained as bipyramidal crystals by recrystallization from water and exo-1,4-epoxy-2-formamido-1,2,3,4tetrahydro-6-trifluoromethylnaphthalene (2) was recrystallized as needles from water-ethanol $(5: 1$ ratio). The crystal dimensions of (1) were $0.2 \times 0.2 \times$ $0.3 \mathrm{~mm}$ and of (2) were $0.2 \times 0.2 \times 0.5 \mathrm{~mm}$. The range of $h k l$ for (1) was $-9 \rightarrow 9,-21 \rightarrow 21,0 \rightarrow 12$ and for (2) was $0 \rightarrow 9,0 \rightarrow 28,0 \rightarrow 5$. Crystals of (1) and (2) were mounted on glass fibers with $\mathbf{c}$ close to the $\varphi$ axis of the diffractometer. Diffraction data out to $2 \theta_{\max }=45 \cdot 0^{\circ}$ were collected using a Syntex $P 2_{1}$ diffractometer (Mo $K \alpha$, graphite monochromator) for (1) and a Rigaku AFC5R (Mo $K \alpha$, graphite monochromator) for (2). Cell constants were determined using $15[(1)]$ and $25[(2)]$ centered reflec. tions widely scattered throughout the space 25 through $35^{\circ}$ in $2 \theta$. The scanning technique for (1) and (2) was $\theta-2 \theta\left(2 \theta>25^{\circ}\right)$. The total number of reflections was 4889 for (1) and 932 for (2). Corrections for crystal decay were made by monitoring two reflections for every 100 measurements [maximum decay correction was 1.011 for (1) and no decay correction was applied for (2)]. Independent $F_{o}^{2}$ data were obtained by merging equivalent reflections. $R_{m}$ $=\sum_{i}^{m} \sum_{i=1}^{n_{j}}\left|I_{i j}-\langle I\rangle_{j}\right| / n_{j} \sum_{j=1}^{m}\langle I\rangle_{j}$ for (1) is 0.035 and for (2) the unique reflections were collected so no $R_{m}$ was calculated. The structure of (1) was solved by direct methods using MULTAN78 (Main, Hull, Lessinger, Germain, Declercq \& Woolfson, 1978) and all calculations were performed on a Honeywell 66/6000 computer at the University of Kansas using programs in the KUDNA system (Takusagawa, 1984). The structure of (2) was solved by direct methods using MITHRIL (Gilmore, 1984) and DIRDIF (Beurskens, 1984) and calculations were performed using the TEXSAN software package (Molecular Structure Corporation, 1985) on a Digital VAX station II/GPX. The function minimized was $\sum w\left(\left|F_{o}\right|-\left|F_{c}\right|\right)^{2}$ with $w=1 / \sigma^{2}\left(F_{o}\right)$. The maximum $\Delta / \sigma$ of the last cycle of least squares was less than 0.26 for (1) and 0.03 for (2) and the minimum value for both was 0.01 . The maximum value for goodness of fit $(S)$ for (1) was 1.22 and for (2) was 1.50. The minimum value $(S)$ for both (1) and (2) was 0.01 . The non-H atoms were refined anisotropically. Positional and isotropic thermal parameters of the $\mathrm{H}$ atoms of compound (1) were refined, while the $\mathrm{H}$ atoms of compound (2) were included in the structure-factor calculation in idealized positions. The final cycle of full-matrix least-squares refinement was based on all independent 1481 reflections and all independent 258 variable parameters for (1) and all independent 910 reflections and 190 variable parameters for (2) and converged with unweighted and weighted agreement factors of $R=0.047$ and $w R=$ 0.051 for (1) and $R=0.108$ and $w R=0.075$ for (2). The refined secondary-extinction value for (1) was 0.738 . For (2) any extinction effects were neglected. The $\mathrm{CF}_{3}$ groups in both compounds were found to be disordered around $\mathrm{C}(8)-\mathrm{C}(13)$ in (1) and $\mathrm{C}(6)$ $\mathrm{C}(13)$ in (2). The groups were refined using a threefold disordered model for (1) and a twofold disordered model for (2). The atom scattering factors were taken from International Tables for $X$-ray Crystallography (1974, Vol. IV). $(\Delta \rho)_{\max },(\Delta \rho)_{\min }=0.31$, -0.33 e $\AA^{-3}$ for (1) and $0.44,-0.42$ e $\AA^{-3}$ for (2).

Discussion. Fractional coordinates and equivalent isotropic thermal parameters for non-hydrogen atoms are given in Table 1 for (1) and (2). Bond lengths, bond angles and torsion angles are shown in 
Table 1. Fractional coordinates and equivalent isotropic thermal parameters for non- $\mathrm{H}$ atoms

$$
B_{\text {eq }}=\left(8 \pi^{2} / 3\right) \sum_{i} \sum_{j} U_{i j} a_{i}^{*} a_{j}^{*} \mathbf{a}_{i} \cdot \hat{a}_{i} .
$$

$\begin{array}{cccc}x & y & z & B\left(\AA^{2}\right)\end{array}$

Compound (1)

$\begin{array}{ll}\mathrm{C}(1) & 0.9700(2) \\ \mathrm{C}(2) & 0.8890(2) \\ \mathrm{C}(3) & 0.7385(3) \\ \mathrm{C}(4) & 0.7625(3) \\ \mathrm{C}(4 \mathrm{a}) & 0.8772(2) \\ \mathrm{C}(5) & 0.8682(3) \\ \mathrm{C}(6) & 0.9980(3) \\ \mathrm{C}(7) & 1.1355(3) \\ \mathrm{C}(8) & 1.1433(2) \\ \mathrm{C}(8 \mathrm{a}) & 1.0125(2) \\ \mathrm{O}(9) & 0.8487(2) \\ \mathrm{N}(10) & 0.8704(2) \\ \mathrm{C}(11) & 0.9791(3) \\ \mathrm{O}(12) & 1.1045(2) \\ \mathrm{C}(13) & 1.2896(2) \\ \mathrm{F}(14 a) & 1.3966(4) \\ \mathrm{F}(15 a) & 1.3420(6) \\ \mathrm{F}(16 a) & 1.2843(5) \\ \mathrm{F}(14 b) & 1.4057(3) \\ \mathrm{F}(15 b) & 1.3071(8) \\ \mathrm{F}(16 b) & 1.3095(8) \\ \mathrm{F}(14 c) & 1.3683(9) \\ \mathrm{F}(15 c) & 1.3778(8) \\ \mathrm{F}(16 c) & 1.2764(6) \\ \end{array}$

$0.3647(1)$
$0.4325(1)$
$0.4130(1)$
$0.3388(1)$
$0.3321(1)$
$0.3180(1)$
$0.3174(1)$
$0.3319(1)$
$0.3493(1)$
$0.3481(1)$
$0.31880(7)$
$0.46181(9)$
$0.4964(1)$
$0.5030(1)$
$0.3664(1)$
$0.3771(5)$
$0.3183(2)$
$0.4212(3)$
$0.3450(6)$
$0.3401(6)$
$0.4319(2)$
$0.4096(6)$
$0.3134(2)$
$0.3944(7)$

$0.4110(2)$
$0.4137(2)$
$0.4637(2)$
$0.4848(2)$
$0.5711(1)$
$0.6777(2)$
$0.7343(2)$
$0.6888(2)$
$0.5826(2)$
$0.5233(2)$
$0.3933(1)$
$0.3090(2)$
$0.2647(2)$
$0.3016(2)$
$0.5347(1)$
$0.6059(3)$
$0.4722(5)$
$0.4755(7)$
$0.5914(6)$
$0.4391(5)$
$0.523(1)$
$0.5922(7)$
$0.521(1)$
$0.4400(5)$

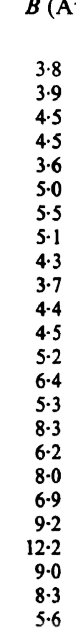

Compound (2)

$\begin{array}{lr}\mathrm{C}(1) & -0.2159(9) \\ \mathrm{C}(2) & -0.3700(8) \\ \mathrm{C}(3) & -0.3784(8) \\ \mathrm{C}(4) & -0.230(1) \\ \mathrm{C}(4 a) & -0.0939(8) \\ \mathrm{C}(5) & 0.0073(9) \\ \mathrm{C}(6) & 0.1231(8) \\ \mathrm{C}(7) & 0.1353(8) \\ \mathrm{C}(8) & 0.0260(7) \\ \mathrm{C}(8 \mathrm{a}) & -0.0850(8) \\ \mathrm{O}(9) & -0.2209(6) \\ \mathrm{N}(10) & -0.5046(7) \\ \mathrm{C}(11) & -0.5984(9) \\ \mathrm{O}(12) & -0.5828(6) \\ \mathrm{C}(13) & 0.234(1) \\ \mathrm{F}(14 a) & 0.349(2) \\ \mathrm{F}(15 a) & 0.322(1) \\ \mathrm{F}(16 a) & 0.169(1) \\ \mathrm{F}(14 b) & 0.210(3) \\ \mathrm{F}(15 b) & 0.357(2) \\ \mathrm{F}(16 b) & 0.201(2)\end{array}$

$0.833(1)$

$0.731(1)$

$0.901(2)$

$1.068(1)$

0.887 (2)

$0.836(2)$

0.647 (2)

0.504 (2)

0.540 (1)

$0.731(1)$
$1.107(1)$

$1.107(1)$
$0.788(1)$

$0.788(1)$
$0.596(2)$

0.360 (1)

0.595 (2)

0.423 (3)

0.803 (2)

$0.523(4)$
$0.776(4)$

$0.776(4)$
$0.601(7)$

0.374 (3)
Table 2. Bond distances $(\AA)$, bond angles $\left({ }^{\circ}\right)$ and torsion angles $\left({ }^{\circ}\right)$ with e.s.d.' $s$ in parentheses for compounds (1), (2)

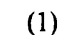

$\mathrm{C}(1)-\mathrm{C}(2)$
$\mathrm{C}(4 a)-\mathrm{C}(5)$
$\mathrm{C}(1)-\mathrm{O}(9)$
$\mathrm{C}(5)-\mathrm{C}(6)$
$\mathrm{C}(2)-\mathrm{C}(3)$
$\mathrm{C}(6)-\mathrm{C}(7)$
$\mathrm{C}(3)-\mathrm{C}(4)$
$\mathrm{C}(8)-\mathrm{C}(8 \mathrm{a})$
$\mathrm{C}(4)-\mathrm{C}(4 a)$
$\mathrm{C}(4)-\mathrm{O}(9)$
$\mathrm{C}(13)-\mathrm{F}(16 c)$
$\mathrm{C}(13)-\mathrm{F}(14 a)$
$\mathrm{C}(13)-\mathrm{F}(14 b)$
$\mathrm{C}(13)-\mathrm{F}(15 b)$
$\mathrm{C}(1)-\mathrm{C}(8 \mathrm{a})$
$\mathrm{C}(13)-\mathrm{C}(6)$
$\mathrm{C}(4 a)-\mathrm{C}(8 \mathrm{a})$
$\mathrm{C}(2)-\mathrm{N}(10)$
$\mathrm{C}(7)-\mathrm{C}(8)$
$\mathrm{C}(8)-\mathrm{C}(13)$
$\mathrm{N}(10)-\mathrm{C}(11)$
$\mathrm{C}(11)-\mathrm{O}(12)$
$\mathrm{C}(13)-\mathrm{F}(15 a)$
$\mathrm{C}(13)-\mathrm{F}(16 a)$
$\mathrm{C}(13)-\mathrm{F}(16 b)$
$\mathrm{C}(13)-\mathrm{F}(15 c)$
$\mathrm{C}(13)-\mathrm{F}(14 c)$

$1.533(3)$

$1.380(3)$

$1.433(3)$

$1.359(4)$
$1.532(3)$

$1.532(3)$

1.383 (4)

$1.521(3)$

$1.385(3)$

$1 \cdot 501(3)$
$1.445(3)$

$1.445(3)$
$1.328(8)$

$1.328(4)$

$1.328(6)$

$1.328(8)$

$1 \cdot 508(3)$

$1 . \overline{384}$ (3)

1.458 (3)

1.390 (3)

$1.476(2)$

$1.314(3)$

$1.328(6)$

$1.327(7)$

$1.327(7)$
$1.328(4)$

$1.328(4)$
$1.327(6)$

1.33 (1)

$\mathrm{C}(2)-\mathrm{C}(1)-\mathrm{C}(8 \mathrm{a})$

$C(1)-C(2)-C(3)$
$C(2)-C(3)-C(4)$

$\mathrm{C}(4 \mathrm{a})-\mathrm{C}(4)-\mathrm{O}(9)$

$\mathrm{C}(5)-\mathrm{C}(4 \mathrm{a})-\mathrm{C}(8 \mathrm{a})$

$\mathrm{C}(6)-\mathrm{C}(7)-\mathrm{C}(8)$

$\mathrm{C}(8 \mathrm{a})-\mathrm{C}(8)-\mathrm{C}(13)$

$\mathrm{C}(4 \mathrm{a})-\mathrm{C}(8 \mathrm{a})-\mathrm{C}(8)$

$\mathrm{N}(10)-\mathrm{C}(11)-\mathrm{O}(12)$

$\mathrm{C}(8)-\mathrm{C}(13)-\mathrm{F}(16 a)$

$C(8)-C(13)-F(16 b)$

$C(8)-C(13)-F(16 c)$

$\mathrm{F}(15 a)-\mathrm{C}(13)-\mathrm{F}(16 b)$

$\mathrm{F}(15 c)-\mathrm{C}(13)-\mathrm{F}(16 \mathrm{c})$

$\mathrm{C}(3)-\mathrm{C}(2)-\mathrm{N}(10)$

$\mathrm{C}(4)-\mathrm{C}(4 \mathrm{a})-\mathrm{C}(8 \mathrm{a})$

$\mathrm{C}(7)-\mathrm{C}(8)-\mathrm{C}(13)$

$C(2)-C(1)-O(9)$

$\mathrm{C}(1)-\mathrm{C}(2)-\mathrm{N}(10)$

$C(3)-C(4)-C(4 a)$

$C(4)-C(4 a)-C(5)$

$C(4 a)-C(5)-C(6)$

$C(7)-C(8)-C(8 a)$

$C(1)-C(8 a)-C(4 a)$

Table 2 for (1) and (2).* Diagrams of the structures are shown in Fig. 1.

The crystal structures of norepinephrine (Carlstrom \& Bergin, 1967) and epinephrine (Andersen, 1975) indicated that the ethanolamine side chain exists in a fully extended conformation. The side chains in our conformationally defined analogs (1) and (2), $\mathrm{N}(10)-\mathrm{C}(2)-\mathrm{C}(1)-\mathrm{C}(8 \mathrm{a})$, also exist in an extended conformation. The torsion angle $\mathrm{N}(10)-\mathrm{C}(2)-\mathrm{C}(1)-\mathrm{C}(8 \mathrm{a})$ is $167 \cdot 42(17)^{\circ}$ for $(1)$ and $172.5(6)^{\circ}$ for $(2)$. The corresponding torsion angle in norepinephrine is $179^{\circ}$ and in epinephrine is $172^{\circ}$. The formamido group in both (1) and (2) is exo. The

* Lists of complete structure factors, anisotropic thermal parameters and $\mathrm{H}$-atom coordinates have been deposited with the British Library Document Supply Centre as Supplementary Publication No. SUP 53327 ( 39 pp.). Copies may be obtained through The Technical Editor, International Union of Crystallography, 5 Abbey Square, Chester CH1 2HU, England.

$\mathrm{C}(1)-\mathrm{C}(8 \mathrm{a})-\mathrm{C}(4 \mathrm{a})$
$\mathrm{C}(1)-\mathrm{O}(9)-\mathrm{C}(4)$

$\mathrm{C}(8)-\mathrm{C}(13)-\mathrm{F}(14 a)$

$\mathrm{C}(8)-\mathrm{C}(13)-\mathrm{F}(14 b)$

$\mathrm{C}(8)-\mathrm{C}(13)-\mathrm{F}(14 c)$

$\mathrm{F}(14 a)-\mathrm{C}(13)-\mathrm{F}(15 a)$

$F(14 b)-C(13)-F(15 b)$

$\mathrm{F}(14 c)-\mathrm{C}(13)-\mathrm{F}(15 c)$

$\mathrm{C}(8 \mathrm{a})-\mathrm{C}(1)-\mathrm{O}(9)$

$\mathrm{C}(3)-\mathrm{C}(4)-\mathrm{O}(9)$

$\mathrm{C}(5)-\mathrm{C}(6)-\mathrm{C}(7)$

$\mathrm{C}(1)-\mathrm{C}(8 \mathrm{a})-\mathrm{C}(8)$

$\mathrm{C}(2)-\mathrm{N}(10)-\mathrm{C}(11)$
$\mathrm{C}(8)-\mathrm{C}(13)-\mathrm{F}(15 b)$

$\mathrm{F}(14 a)-\mathrm{C}(13)-\mathrm{F}(16 a)$

$F(14 c)-C(13)-F(16 c)$

$\mathrm{C}(5)-\mathrm{C}(6)-\mathrm{C}(13)$

$\mathrm{C}(6)-\mathrm{C}(13)-\mathrm{F}(16 a)$

$\mathrm{C}(6)-\mathrm{C}(13)-\mathrm{F}(16 b)$

$\mathrm{C}(6)-\mathrm{C}(13)-\mathrm{F}(14 b)$

$C(8)-C(13)-F(15 a)$

$\mathrm{C}(8)-\mathrm{C}(13)-\mathrm{F}(15 c)$

$\mathrm{F}(14 b)-\mathrm{C}(13)-\mathrm{F}(16 b)$

$\mathrm{C}(7)-\mathrm{C}(6)-\mathrm{C}(13)$

$C(6)-C(13)-F(15 b)$

C(6)-C(13)-F(15a)

$C(8 a)-C(1)-C(2)-C(3)$

$O(9)-C(1)-C(2)-C(3)$
106.9 (2)

$101 \cdot 2(2)$

$101.4(2)$
$101.4(2)$

$121.7(2)$

$121.7(2)$

$120.0(2)$

$121.5(2)$

124.6 (3)

$113.0(3)$

$113 \cdot 0$ (4)

$113.0(3)$

$105 \cdot 8$ (4)

$105 \cdot 8(8)$

$105.9(8)$

$112 \cdot 2(2)$

$104 \cdot 6(2)$
$120.0(2)$

$102 \cdot 4(2)$

$112.8(2)$

$108.0(2)$

133.6 (2)

117.9 (3)

$118.5(2)$

$104 \cdot 2$ (2)

$95.6(2)$

113.0 (2)

112.9 (4)

112.9 (4)

105.6 (4)

105.9 (5)

106.0 (6)

$101.3(2)$

$101.7(2)$

$122.0(2)$
$135.9(2)$

$135.9(2)$
$121.0(2)$

112.8 (4)

106.0 (5)

105.5 (7)

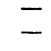

-

112.8 (3)

112.9 (4)

$105.8(7)$

$\begin{array}{cc}105.8(7) & 97(1) \\ = & 119.3(8) \\ = & 111(1) \\ - & 112.7(9) \\ -72.4(2) & 115.9(8) \\ 33.6(2) & -70.7(8) \\ & 35.9(6)\end{array}$

1.54 (1)

1.378 (9)

$1.43(2)$
$1.38(1)$

1.55 (1)

1.36 (1)

1.52 (1)

1.36 (1)
$1.50(1)$

$1.50(1)$
$1.457(8)$

$1 . \overline{34}$ (1)

$1.44(2)$

$1 \cdot 11$ (2)

1.51 (1)

$1.48(1)$
$1.40(1)$

1.469 (9)

1.414 (9)

1.35 (1)

1.21 (2)

1.27 (1)

$1.27(1)$
$1.33(2)$

-

$106 \cdot 7$ (5)

$100.5(6)$

$102.3(6)$

119.1 (8)

120.6 (7)

$122 \cdot 1$ (7)

127.1 (9)

=

106 (1)

113 (2)

$110.3(6)$

104.9 (7)

$101 \cdot 6(6)$

$111.6(5)$

107.1 (8)

135.9 (7)

119.2 (7)

$117.6(7)$

$104 \cdot 3(7)$
$96.5(5)$

-

99 (1)

111 (2)

102.1 (6)

$100 \cdot 6(6)$

$121 \cdot 1$ (7)

$133.6(6)$

$122 \cdot 4$ (7)

$108(1)$

$119 \cdot 6$ (7)

$114 \cdot 1$ (7)

113 (1)

111 (1)

97 (1)

(1)

12.7 (9)

$70.7(8)$
$35.9(6)$ 
Table 2 (cont.)

\begin{tabular}{|c|c|c|}
\hline & (1) & $(2)$ \\
\hline$C(2)-C(1)-C(8 a)-C(4 a)$ & $72 \cdot 1(2)$ & $74 \cdot 1(8)$ \\
\hline$C(2)-C(1)-O(9)-C(4)$ & $-56 \cdot 8(2)$ & $-58.8(6)$ \\
\hline$C(1)-C(2)-C(3)-C(4)$ & $2 \cdot 3(2)$ & $-0.1(8)$ \\
\hline$C(1)-C(2)-N(10)-C(11)$ & $-83.4(3)$ & $-121 \cdot 2(7)$ \\
\hline$C(2)-C(3)-C(4)-C(4 a)$ & $69 \cdot 1(2)$ & $70.8(7)$ \\
\hline$C(3)-C(4)-C(4 a)-C(5)$ & $102 \cdot 4(3)$ & $104(1)$ \\
\hline$O(9)-C(4)-C(4 a)-C(5)$ & $-151 \cdot 1(2)$ & $-151.4(9)$ \\
\hline$C(3)-C(4)-O(9)-C(1)$ & $58 \cdot 4(2)$ & $58.2(7)$ \\
\hline$C(4)-C(4 a)-C(5)-C(6)$ & $-178 \cdot 5(2)$ & $180(4)$ \\
\hline$C(4)-C(4 a)-C(8 a)-C(1)$ & $1.0(2)$ & $-1 \cdot 1(8)$ \\
\hline$C(4 a)-C(5)-C(6)-C(7)$ & $1.7(4)$ & $2(1)$ \\
\hline$c(6)-C(7)-C(8)-C(8 a)$ & $-3.5(4)$ & $-4(1)$ \\
\hline$C(7)-C(8)-C(8 a)-C(1)$ & $177.6(2)$ & $-177 \cdot 8(7)$ \\
\hline$C(13)-C(8)-C(8 a)-C(1)$ & $-4.5(4)$ & - \\
\hline $\mathrm{C}(7)-\mathrm{C}(8)-\mathrm{C}(13)-\mathrm{F}(14 a)$ & $-13.8(5)$ & - \\
\hline$C(2)-N(10)-C(11)-O(12)$ & $5 \cdot 4(4)$ & $4(1)$ \\
\hline$C(8 a)-C(8)-C(13)-F(16 c)$ & $14 \cdot 6(6)$ & - \\
\hline $\mathrm{O}(9)-\mathrm{C}(1)-\mathrm{C}(8 \mathrm{a})-\mathrm{C}(4 \mathrm{a})$ & $-34.8(2)$ & $-32 \cdot 1(7)$ \\
\hline$C(8 a)-C(8)-C(13)-F(16 b)$ & $80 \cdot 2(7)$ & - \\
\hline$C(8 a)-C(8)-C(13)-F(14 b)$ & $-159.8(6)$ & - \\
\hline $\mathrm{C}(8 \mathrm{a})-\mathrm{C}(8)-\mathrm{C}(13)-\mathrm{F}(15 a)$ & $-71.9(4)$ & \\
\hline$C(7)-C(8)-C(13)-F(16 c)$ & $-167 \cdot 5(6)$ & - \\
\hline $\mathrm{C}(7)-\mathrm{C}(8)-\mathrm{C}(13)-\mathrm{F}(15 c)$ & $72.4(7)$ & - \\
\hline$C(7)-C(8)-C(13)-F(14 c)^{\prime}$ & $-47.9(6)$ & - \\
\hline$C(7)-C(8)-C(13)-F(16 a)$ & $-134 \cdot 1(4)$ & - \\
\hline$C(5)-C(6)-C(13)-F(14 a)$ & - & $175(1)$ \\
\hline $\mathrm{C}(5)-\mathrm{C}(6)-\mathrm{C}(13)-\mathrm{F}(15 a)$ & - & $63(1)$ \\
\hline $\mathrm{C}(5)-\mathrm{C}(6)-\mathrm{C}(13)-\mathrm{F}(16 a)$ & - & $-59(2)$ \\
\hline $\mathrm{C}(8 \mathrm{a})-\mathrm{C}(1)-\mathrm{C}(2)-\mathrm{N}(10)$ & $167 \cdot 4(2)$ & $172.5(6)$ \\
\hline $\mathrm{O}(9)-\mathrm{C}(1)-\mathrm{C}(2)-\mathrm{N}(10)$ & $-86.6(2)$ & $-81.0(7)$ \\
\hline$C(2)-C(1)-C(8 a)-C(8)$ & $-103.7(3)$ & $-107(1)$ \\
\hline$C(8 a)-C(1)-O(9)-C(4)$ & $53.5(2)$ & $51 \cdot 3(7)$ \\
\hline$N(10)-C(2)-C(3)-C(4)$ & $122.8(2)$ & $117.7(7)$ \\
\hline$C(3)-C(2)-N(10)-C(11)$ & $162 \cdot 9(2)$ & $128.1(8)$ \\
\hline$C(2)-C(3)-C(4)-O(9)$ & $-37 \cdot 1(2)$ & $-34.9(7)$ \\
\hline$C(3)-C(4)-C(4 a)-C(8 a)$ & $-73.7(2)$ & $-71.8(8)$ \\
\hline$O(9)-C(4)-C(4 a)-C(8 a)$ & $32.7(2)$ & $33.2(8)$ \\
\hline$C(4 a)-C(4)-O(9)-C(1)$ & $-52.9(2)$ & $-51.9(7)$ \\
\hline$C(8 a)-C(4 a)-C(5)-C(6)$ & $-2.9(3)$ & $-5(1)$ \\
\hline$C(4)-C(4 a)-C(8 a)-C(8)$ & $177 \cdot 6(2)$ & $-180(2)$ \\
\hline$C(5)-C(6)-C(7)-C(8)$ & $1.5(4)$ & $2(1)$ \\
\hline$C(6)-C(7)-C(8)-C(13)$ & $178.6(2)$ & - \\
\hline$C(7)-C(8)-C(8 a)-C(4 a)$ & $2 \cdot 3(3)$ & $1(1)$ \\
\hline$C(13)-C(8)-C(8 a)-C(4 a)$ & $-179.8(2)$ & - \\
\hline $\mathrm{C}(7)-\mathrm{C}(8)-\mathrm{C}(13)-\mathrm{F}(15 a)$ & $105.9(4)$ & - \\
\hline$C(8 a)-C(8)-C(13)-F(16 c)$ & $14.6(6)$ & - \\
\hline$C(8 a)-C(8)-C(13)-F(15 c)$ & $-105.5(7)$ & - \\
\hline$C(8 a)-C(8)-C(13)-F(14 c)$ & $134 \cdot 2(5)$ & - \\
\hline$C(8 a)-C(8)-C(13)-F(15 b)$ & $-39.8(6)$ & - \\
\hline $\mathrm{C}(8 \mathrm{a})-\mathrm{C}(8)-\mathrm{C}(13)-\mathrm{F}(16 a)$ & 48.0 (4) & - \\
\hline$C(8 a)-C(8)-C(13)-F(14 a)$ & $168 \cdot 3(5)$ & - \\
\hline$C(7)-C(8)-C(13)-F(16 b)$ & $-101.9(7)$ & - \\
\hline $\mathrm{C}(7)-\mathrm{C}(8)-\mathrm{C}(13)-\mathrm{F}(15 b)$ & $138 \cdot 1(6)$ & - \\
\hline$C(7)-C(8)-C(13)-F(14 h)$ & $18 \cdot 1(6)$ & - \\
\hline $\mathrm{C}(7)-\mathrm{C}(8)-\mathrm{C}(13)-\mathrm{F}(14 a)$ & - & $-6(1)$ \\
\hline $\mathrm{C}(7)-\mathrm{C}(8)-\mathrm{C}(13)-\mathrm{F}(15 a)$ & - & $-118(1)$ \\
\hline $\mathrm{C}(7)-\mathrm{C}(8)-\mathrm{C}(13)-\mathrm{F}(16 a)$ & - & $120(1)$ \\
\hline$C(7)-C(8)-C(13)-F(14 b)$ & - & $-176(1)$ \\
\hline$C(5)-C(6)-C(13)-F(14 b)$ & - & $5(2)$ \\
\hline $\mathrm{C}(5)-\mathrm{C}(6)-\mathrm{C}(13)-\mathrm{F}(15 b)$ & - & $129(2)$ \\
\hline$C(5)-C(6)-C(13)-F(16 b)$ & - & $-103(1)$ \\
\hline$C(7)-C(8)-C(13)-F(15 b)$ & - & $-52(2)$ \\
\hline$C(7)-C(8)-C(13)-F(16 b)$ & - & $76(1)$ \\
\hline
\end{tabular}

$\mathrm{X}$-ray studies on (1) suggest the presence of a hydrogen bond between $\mathrm{N}(10)$ and $\mathrm{O}(12) \quad \mathrm{N}(10)-$ $\mathrm{H}(10) \cdots \mathrm{O}(12)]$. The distance between $\mathrm{N}(10)$ and $\mathrm{O}(12)$ is 2.837 (3) $\AA$. The distance between $\mathrm{H}(10)$ and $\mathrm{O}(12)$ is $1.98(3) \AA$. The bond angle $\mathrm{N}(10)-$ $\mathrm{H}(10) \cdots \mathrm{O}(12)$ is $160(2)^{\circ}$. Similarly the studies on (2) also suggest the presence of a hydrogen bond between $\mathrm{N}(10)$ and $\mathrm{O}(12)$. The distance between $\mathrm{N}(10)$ and $\mathrm{O}(12)$ is 2.99 (3) $\AA$. The distance between $\mathrm{N}(10)$ and $\mathrm{H}(10)$ is $0.949 \AA$. These studies do not support the presence of a hydrogen bond between $\mathrm{NH}$ and $\mathrm{F}$ atoms.

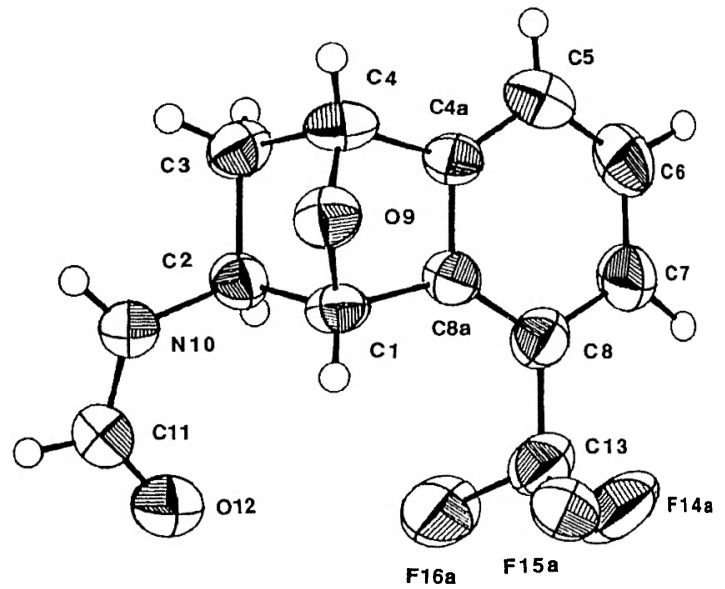

(1)

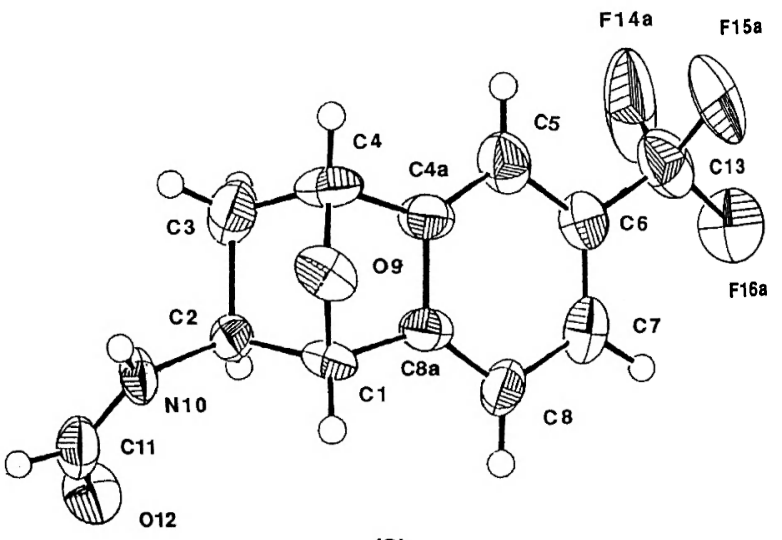

(2)

Fig. 1. ORTEPII (Johnson, 1976) drawings of (1) and (2). H atoms are displayed as small spheres of arbitrary size and non- $\mathrm{H}$ atoms as principal ellipses at the $50 \%$ probability level.

Biochemical results comparing analogs [(4)-(7)] $\left(X=-\mathrm{CH}_{2}-\right.$ and $X=-\mathrm{O}-$ ) (Palanki, Wong, Criscione \& Grunewald, 1988) show a similar regiochemical preference of the $\mathrm{CF}_{3}$ group with analog (6) being a good substrate for PNMT with $X=-\mathrm{CH}_{2}-$ or $X=-\mathrm{O}$. Since the addition of the oxygen bridge lowers the lipid-aqueous partition coefficient $\left[\log P\right.$ of (4) $\left(X=-\mathrm{CH}_{2}-\right)=3 \cdot 20 ; \log P$ of $(4)(X=$ $-\mathrm{O}-)=1.86 ; \quad$ (Grunewald, Pleiss, Gatchell, Pazhenchevsky \& Rafferty, 1984)], it appears that $\mathrm{CF}_{3}$ group is binding interaction provided by the $\mathrm{CF}_{3}$ group is a regional effect due to interaction at the aromatic-ring binding site and is not due to the overall lipophilicity of the entire molecule.

This work was supported by the National Institutes of Health Grant HL 34193.

\footnotetext{
References

ANDERSEN, A. M. (1975). Acta Chem. Scand. Ser. B, 29, 239-
244.
} 
Beurskens, P. T. (1984). DIRDIF. Tech. Rep. 1984/1. Crystallography Laboratory, Toernooiveld, 6525 ED Nijmegen, The Netherlands.

Carlstrom, D. \& Bergin, R. (1967). Acta Cryst. 23, 313-319.

Gilmore, C. J. (1984). J. Appl. Cryst. 17, 42-46.

Grunewald, G. L., Arrington, H. S., Bartlett, W. J., Reitz, T. J. \& SALl, D. J. (1986). J. Med. Chem. 29, 1972-1982.

Grunewald, G. L., Paradkar, V. M., Pazhenchevsky, B., Pleiss, M. A., Sall, D. J., Seibel, W. L. \& Reitz, T. J. (1983). J. Org. Chem. 48, 2321-2327.

Grunewald, G. L., Pleiss, M. A., Gatchell, C. I., PAZHenchevsky, B. \& Rafferty, M. F. (1984). J. Chromatogr. 292, 319-324.

Grunewald, G. L., Ye, Q. \& Takusagawa, F. (1987). Acta Cryst. C43, 2418-2420.

JoHnson, C. K. (1976). ORTEPII. Report ORNL-5138. Oak Ridge National Laboratory, Tennessee, USA.
Main, P., Hull, S. E., Lessinger, L., Germain, G., Declerce, J.-P. \& Woolfson, M. M. (1978). MULTAN78. A System of Computer Programs for the Automatic Solution of Crystal Structures from $X$-ray Diffraction Data. Univs. of York, England, and Louvain, Belgium.

Molecular Structure Corporation (1985). TEXSAN. TEXRAY Structure Analysis Package. MSC, 3200A Research Forest Drive, The Woodlands, TX77381, USA.

Palanki, M. S. S., Wong, N., Criscione, K. \& Grunewald, G. L. (1988). 23rd Midwest Regional Meeting of the American Chemical Society, Iowa City, Iowa, 16-18 November. Abstract No. 071. To be published.

Takusagawa, F. (1984). DUDNA. Unpublished work. Department of Chemistry, Univ. of Kansas, Lawrence, Kansas 66045, USA.

Ye, Q. \& Grunewald, G. L. (1989). J. Med. Chem. 32, 478486.

Acta Cryst. (1991). C47, 775-777

\title{
Structure of 3-(Bromoacetyl)coumarin
}

\author{
By Krishnagiri T. Vasudevan and Puttaraja \\ Department of Physics, Bangalore University, Bangalore-560056, India \\ AND MANOHAR V. KULKaRNI \\ Department of Chemistry, Central College, Bangalore University, Bangalore-560001, India
}

(Received 19 March 1990; accepted 28 June 1990)

\begin{abstract}
C}_{11} \mathrm{H}_{7} \mathrm{BrO}_{3}, \quad M_{r}=267 \cdot 09$, monoclinic, $P 2_{1} / n, a=21.555(2), b=4 \cdot 229(2), c=10.784$ (1) $\AA$, $\beta=93.89(1)^{\circ}, \quad V=980.8 \AA^{3}, Z=4, D_{m}=1.79(5)$, $D_{x}=1.808 \mathrm{Mg} \mathrm{m}^{-3}, \quad \lambda(\mathrm{Cu} \mathrm{K \alpha})=1.5418 \AA, \quad \mu=$ $5 \cdot 2 \mathrm{~mm}^{-1}, F(000)=528, T=300 \mathrm{~K}$. Final $R$ for 1692 observed reflections is 0.054 . The coumarin moiety is nearly planar and makes a dihedral angle of $4.5(8)^{\circ}$ with the mean plane of the bromoacetyl group. The molecule adopts an $S$-cis eclipsed conformation in the solid state and the molecules are linked by weak $\mathrm{C}-\mathrm{H} \cdots \mathrm{O}$ bonds.
\end{abstract}

Introduction. The coumarin moiety with various groups at the 3 position has given rise to many derivatives of biological and structural importance. 3-(Bromoacetyl)coumarin (Koelsch, 1950) has been a key intermediate in the synthesis of some 3heterocyclic coumarins (Kulkarni, Patil, Biradar \& Nanjappa, 1981) and many other compounds of biological relevance (Hanumanthgad, Kulkarni \& Patil, 1984). A preliminary examination of its structure shows that due to free rotation about the $\mathrm{C}(3)-\mathrm{CO}$ bond this molecule can give rise to two conformations. In these two conformers the carbonyl group attached to C(3) can be $S$-cis (I) or $S$-trans (II) to the $\mathrm{C}(3)-\mathrm{C}(4)$ double bond of the pyrone ring.

0108-2701/91/040775-03\$03.00
Further, many $o$-haloketones are known to exhibit interesting conformations (Rao, 1963) which have been explained using different projection formulae when the molecule is viewed along the $\mathrm{OC}-\mathrm{CH}_{2} \mathrm{Br}$ axis. In order to establish the preferred conformation of the title compound in the solid state, an X-ray crystal structure analysis has been carried out.<smiles>O=C(CBr)c1cc2ccccc2oc1=O</smiles>

(I)<smiles>O=C(CBr)c1cc2ccccc2oc1=O</smiles>

(II)
Experimental. Colourless transparent crystals grown by slow evaporation from chloroform solution, $D_{m}$ by flotation in a mixture of bromoform and acetone. Crystal of size $0.2 \times 0.3 \times 0.3 \mathrm{~mm}$ mounted on an Enraf-Nonius CAD-4 diffractometer, $\omega-2 \theta$ scan, variable scan rate. Lattice parameters from leastsquares refinement of 25 centred reflections; $\mathrm{Cu} \mathrm{K \alpha}$ intensity data collected to a maximum $2 \theta=152^{\circ}$ $(-26<h<26,0<k<5,0<l<12)$; two standard reflections (711 and 016) monitored at regular intervals, intensity variation $2 \%$. 2184 reflections of 